\title{
PREPARACIÓN Y CARACTERIZACIÓN DE CARBONES ACTIVADOS A PARTIR DE UN CARBÓN MINERAL DE LA CUENCA DEL CESAR (Colombia)
}

\author{
Lina Mercedes Daza Barrancoㄹ, Juan Fernando Orrego Miranda², Jorge Luis Piñeres Mendoza ${ }^{3}$, Jhon \\ Jairo Fernández Hincapie ${ }^{4}$ \\ ${ }^{1}$ Química. Universidad del Atlántico. Grupo de Investigación en Fotoquímica y Fotobiología. Barranquilla- \\ Colombia.Imdaza@mail.uniatlantico.edu.co \\ 2Doctor en Ciencias Químicas. Profesor asistente Universidad del Atlántico. Grupo de Investigación en \\ Fotoquímica y Fotobiología. Barranquilla-Colombia. juanorrego@mail.uniatlantico.edu.co \\ ${ }^{3}$ Doctor en Ingeniería. Universidad del Atlántico. Grupo de Simulación de Procesos y Transformación del \\ Carbón. Barranquilla-Colombia. jorgepineres@mail.uniatlantico.edu.co \\ ${ }^{4}$ Doctor en Química e Ingeniería de Procesos. Profesor Universidad de Antioquia. Grupo de Investigación \\ Química de Recursos Energéticos y Medio Ambiente. Medellín-Colombia.John.fernandez@udea.edu.co
}

\section{RESUMEN}

En este trabajo se prepararon carbones activados a partir de un carbón bituminoso, mediante procesos de activación física y química. La activación física con vapor de agua $\left(\mathrm{H}_{2} \mathrm{O}\right)$ se realizó a temperaturas de 700 y $800^{\circ} \mathrm{C}$ y la activación química utilizando $\mathrm{ZnCl}_{2} 0.2 \mathrm{~N}$ a 600,700 y $800^{\circ} \mathrm{C}$. Los carbones activados se caracterizaron mediante diferentes técnicas, como punto de carga cero (PZC), espectroscopia infrarroja (IR, DRIFT), área superficial y microscopia electrónica de barrido (SEM-EDX). Se obtuvieron carbones activados microporosos con áreas superficiales hasta de $351 \mathrm{~m}^{2} / \mathrm{g}$ con un volumen de poro de $0.15 \mathrm{~cm} / \mathrm{g}$ y un tamaño promedio de poro de 19.4 A. El carbón activado de mayor área superficial fue apto para la remoción del azul de metileno cuya isoterma de adsorción se ajusta al modelo de Langmuir y la cinética de adsorción se ajusta al modelo pseudo-segundo orden y de difusión intraparticular.

Palabras clave: Carbón activado, carbón bituminoso, activación físico-química, propiedades de adsorción, remoción de colorante, cinética

Recibido: 14 de Agosto de $2017 . \quad$ Aceptado: 18 de Junio de 2018

Received: August 17th, $2017 . \quad$ Accepted: June 18th, 2018

\section{PREPARATION AND CHARACTERIZATION OF ACTIVATED CARBONS FROM COAL OF CESAR'S BASIN (Colombia)}

\begin{abstract}
In this work, activated carbons were prepared from a bituminous coal, through physical and chemical activation processes. Physical activation with water vapor $\left(\mathrm{H}_{2} \mathrm{O}\right)$ was performed at temperatures of 700 and $800^{\circ} \mathrm{C}$ and chemical activation using $0.2 \mathrm{~N} \mathrm{ZnCl} 2$ at 600, 700 and $800^{\circ} \mathrm{C}$. Activated carbons were characterized by different techniques, such as zero load point (PZC), infrared spectroscopy (IR, DRIFT), surface area and scanning electron microscopy (SEM-EDX). Microporous activated carbons were obtained with surface areas up to $351 \mathrm{m2} / \mathrm{g}$ with a pore volume of $0.15 \mathrm{~cm} 3 / \mathrm{g}$ and a mean pore size of $19.4 \AA$. The activated carbon of greater surface area was suitable for the removal of the methylene blue whose adsorption isotherm fits to the Langmuir model and the kinetics of adsorption is adjusted to the pseudo-second order and intraparticular diffusion model.
\end{abstract}

Keywords: Activated carbon, bituminous coal, physical-chemical activation, adsorption properties, dye removal, kinetics.

Cómo citar este artículo: L. M. Daza Barranco, J. F. Orrego Miranda, J. L. Piñeres Mendoza, J. J. Fernández Hincapie, "Preparación y Caracterización de Carbones Activados a partir de un Carbón Mineral de la Cuenca del Cesar (Colombia)", Revista Politécnica, Año 14, Vol 14, No 26, pp 75-88, 2018.

https://doi.org/10.33571/rpolitec.v14n26a7 


\section{INTRODUCCIÓN}

En los últimos años, el aumento en la contaminación del medio ambiente ha obligado a establecer límites en la calidad del agua que se vienten en los efluentes líquidos. Un grupo importante dentro de los contaminantes presentes son los compuestos orgánicos (CO) y se encuentran presentes debido a sus extensos usos como solventes y reactivos en muchas industrias. La Organización Mundial de la Salud (OMS) ha alertado sobre la toxicidad de estos CO. Muchos de ellos se encuentran en vertimientos generados por la actividad industrial desarrollada por el hombre, que contienen compuestos tóxicos en concentraciones altas, que constituyen así una gran fuente de contaminación afectando la flora, la fauna y la salud humana.[1] Uno de los principales problemas de los residuos peligrosos es el impacto en la incapacidad del medio ambiente para asimilarlos. La eliminación de estos contaminantes en los efluentes líquidos es de gran importancia, en el cual los métodos utilizados para estos procesos deben ser económicos, fáciles de manipular, no formar productos secundarios tóxicos y no incitar en alteraciones ecológicas.[2, 3]

Los colorantes son muy utilizados en la industria del cuero, agrícola y alimentaria, entre otro. Pero su mayor consumo se da en la industria de textiles y de artes gráficas. El tratamiento de vertimientos que contienen colorantes presentan gran dificultad debido a que éstos son compuestos resistentes a la digestión aerobia, a los agentes oxidantes convencionales y a los tratamientos térmicos. Es por esta razón que en las últimas décadas se han intentado desarrollar tratamientos más eficaces biológicos, físicos y químicos, para la decoloración de éstas aguas.[4]

En la actualidad, la adsorción es considerada como una de las mejores técnicas de remoción de contaminantes. Entre los adsorbentes se enfatizan materiales porosos como el carbón activado (CA) debido a su gran área superficial, su química superficial y su estructura porosa, propiedades que le confieren una buena capacidad de adsorción para remover diversos contaminantes de agua y en corrientes gaseosas, que contienen pesticidas, iones metálicos, micro-contaminantes orgánicos, entre otros.[5, 6] El proceso de adsorción con materiales porosos y de elevada área superficial ha resultado el método predominante debido principalmente por su bajo costo inicial, versatilidad, insensibilidad a sustancias tóxicas, simplicidad del diseño y facilidad de operación.[7]

El CA es un adsorbente que se puede preparar a partir de materiales carbonosos y se caracteriza por poseer una alta superficie interna, una buena distribución de poros y variedad de grupos funcionales, propiedades que le permiten interaccionar favorablemente con gran diversidad de moléculas.[8]

Es por eso que el presente trabajo se orientó hacia la obtención de un CA y el estudio de los procesos de adsorción sobre el mismo. Para esto se empleó el colorante azul de metileno como compuesto modelo debido a que este es un colorante ampliamente manipulado como indican los numerosos reportes donde se aborda el estudio del fenómeno de adsorción sobre distintos materiales.

El objetivo de este trabajo fue utilizar carbón mineral de Colombia para preparar carbones activados por activación física con vapor de agua y química con cloruro de zinc, con el fin de darle un valor económico al carbón mineral. Cabe destacar que el beneficio de emplear el carbón mineral para generar carbón activado, se debe a que en Colombia los niveles de consumo de carbón son medianos comparados con la abundancia de reservas, la distribución geográfica de las cuencas carboníferas y el potencial identificado para su uso, lo cual sería provechoso para la economía, reduciendo los costos elevados que se emplean anualmente en el control de la contaminación ambiental de nuestro país. Además se caracterizaron los carbones activados con análisis próximos, elementales, área superficial, distribución del tamaño del poro, microscopia electrónica SEMEDX, espectroscopia de infrarrojo FTIR y DRIFT, punto de carga cero.[9]

\section{MATERIALES Y METODOS}

Preparación de la muestra y obtención de los carbones activados: Se utilizó como materia prima un carbón mineral bituminoso proveniente del Departamento del Cesar, Colombia. El carbón mineral bituminoso fue macerado y tamizado a un diámetro de partícula de 500 a $1000 \mu \mathrm{m}$. Usando este carbón mineral bituminoso, se prepararon carbones activados con dos diferentes metodologías; activación física con vapor de agua y activación química con cloruro de zinc $0.2 \mathrm{~N}$. 
Activación física: Los carbones activados fueron obtenidos mediante pirólisis del carbón mineral bituminoso y posterior activación del pirolizado. En un horno horizontal con tres zonas de calentamiento HEATECH, (Fig. 1), dentro de una cápsula de acero, fue colocado el carbón mineral pirolizándolo en atmósfera de nitrógeno a $800^{\circ} \mathrm{C}$ por 1 hora. Luego las muestras fueron enfriadas a temperatura ambiente. Posteriormente para la activación, usando el mismo horno, las muestras fueron llevadas hasta la temperatura de activación en atmósfera de nitrógeno, gas que fue cambiado por el agente activante (nitrógeno saturado con vapor de agua a $80^{\circ} \mathrm{C}$ ) evaluando el efecto de diferentes temperaturas y tiempos de activación como se aprecia en la Tabla 1. Finalmente las muestras fueron enfriadas en atmósfera de nitrógeno.

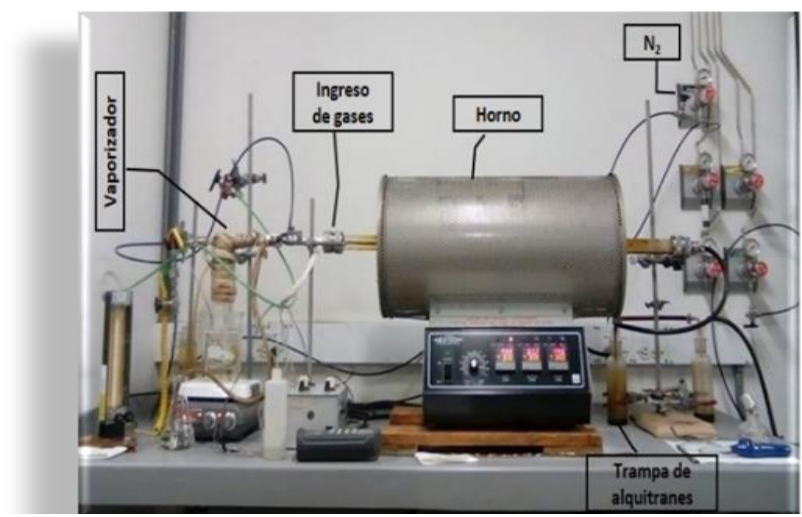

Fig. 1. Horno horizontal HEATECH, propiedad del grupo QUIREMA de la Universidad de Antioquia

Tabla 1. Condiciones para la preparación de los carbones activados físicamente.

\begin{tabular}{lllll}
\hline \multicolumn{1}{c}{ Condiciones } & \multicolumn{2}{c}{$\begin{array}{c}\text { Agente activante } \\
(\mathbf{1 0 0} \mathbf{~} \mathbf{m L} / \mathbf{m i n}) \\
\mathbf{H}_{\mathbf{2}} \mathbf{O} / \mathbf{N}_{\mathbf{2}}\end{array}$} \\
\hline $\begin{array}{l}\text { Temperatura máxima } \\
\left({ }^{\circ} \mathbf{C}\right)\end{array}$ & 700 & 800 \\
$\begin{array}{l}\text { Tiempo de Isoterma } \\
\text { (min) }\end{array}$ & 45 & 90 & 45 & 90 \\
$\begin{array}{l}\text { Nomenclatura de los } \\
\text { carbones }\end{array}$ & CAF_745 & CAF_845 \\
\hline CAF_790 & CAF_890 \\
\hline
\end{tabular}

Activación química: Para la activación química, el carbón mineral bituminoso fue molido y tamizado a $500-1000 \mu \mathrm{m}$, luego fue impregnada con una solución de cloruro de zinc $0.2 \mathrm{~N}$ por un periodo de tiempo de 48 horas a temperatura ambiente, posteriormente la muestra fue secada en estufa a $110^{\circ} \mathrm{C}$. Finalmente, la muestra fue carbonizada a $800^{\circ} \mathrm{C}$ por 1 hora en atmósfera de $\mathrm{N} 2$ y activada en un horno tipo mufla (Fig. 2) a diferentes condiciones como se aprecia en la Tabla 2, después de enfriarse fue lavada con ácido clorhídrico y agua destilada hasta $\mathrm{pH}$ neutro.

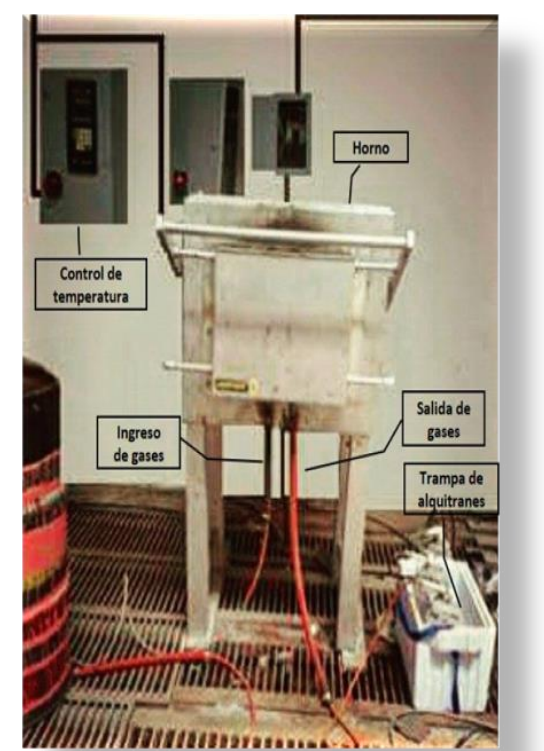

Fig. 2. Horno horizontal propiedad del grupo CARBOQUÍMICA de la Universidad del Atlántico

Tabla 2: Condiciones para la preparación de los carbones activados químicamente.

Agente activante

\section{Condiciones}

(100 mL/min)

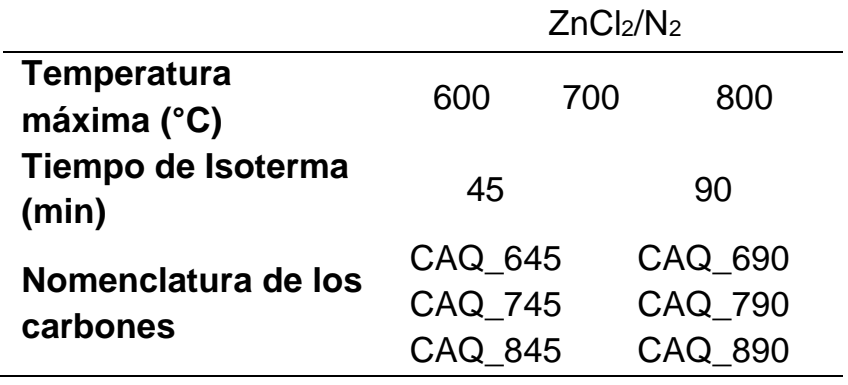

Caracterización de los carbones activados: Para la caracterización del carbón mineral y los carbones activados, se realizaron análisis próximos usando un equipo termogravimétrico TGA SDT-Q600 marca TA Instruments. Los análisis termogravimétricos de los carbones en estudio fueron realizados a una velocidad de calentamiento 
de $20^{\circ} \mathrm{C} /$ minuto en atmósfera de nitrógeno. Finalmente a $900^{\circ} \mathrm{C}$ el nitrógeno fue sustituido por aire, y la combustión del carbono fijo da paso a la formación de cenizas.[10-12]

El análisis de $\mathrm{C}, \mathrm{H}, \mathrm{N}, \mathrm{S}$, y $\mathrm{O}$ fue efectuado en un equipo de análisis elemental (CHNSO) marca LECO, modelo Truspec. El análisis de área superficial fue realizado en un analizador de área superficial y porosidad ASAP 2020 marca Micrometrics.[13, 14] La morfología de las muestras fue evaluada en alto vacío y con las señales de electrones secundarios y retrodispersados, usando un Microscopio Electrónico de Barrido (SEM-EDX) marca JEOL JSM-6490 LV- EDX modelo Oxford Instruments.[15] Los análisis espectrales se realizaron en un equipo infrarrojo Shimadzu FTIR/DRIFT modelo Affinity-1, en un rango espectral $4000-400 \mathrm{~cm}^{-1}$.[16] El punto de carga cero fue medido por el método de balance de masa, empleando en una solución de cloruro de sodio en distintos $\mathrm{pH} .[17]$

Adsorción y cinética de los carbones activados: Se realizó el estudio de adsorción y cinética para el carbón activado con mayor área superficial y porosidad formada, empleando como colorante azul de metileno en diferentes concentraciones (50-1000 $\mathrm{mg} / \mathrm{L}$ ) en $\mathrm{pH} 8$ a temperatura de $298 \mathrm{~K}$, en espectrofotómetro Thermo modelo Genesys 20 UVVis. Para la adsorción se tomó $0.5 \mathrm{~g}$ del carbón activado en $0.05 \mathrm{~L}$ de azul de metileno a distintas concentraciones, luego se toman $5 \mathrm{~mL}$ de la alicuota y se midió la absorbancia a una longitud de onda de $665 \mathrm{~nm}$.[18] Para la cinética en 0.250L de las soluciones de azul de metileno se le adicionaron $0.5 \mathrm{~g}$ del carbón activado, se le realizó la medición de la absorbancia a $665 \mathrm{~nm}$ cada hora durante $12 \mathrm{~h}$ hasta alcanzar el equilibrio a temperatura y agitación constante.[19]

\section{RESULTADOS Y DISCUSIONES}

Rendimiento de los carbones activados: Para los carbones activados obtenidos se calculó el rendimiento porcentual según la ecuación:

$\% R=\left(\frac{m_{C A}}{m_{M P}}\right) \times 100$

En la Fig. 3 se observan los rendimientos de cada una de las muestras preparadas. En general se observa que tanto para la activación física como para la química, el rendimiento disminuye a medida que la temperatura y el tiempo de activación aumentan. Los rendimientos obtenidos para la activación física con vapor de agua se encuentran entre el $55-59 \%$, en cambio se observa un mejor rendimiento en los carbones activados químicamente con cloruro de zinc $(0.2 \mathrm{~N})$ dentro de un rango del $61-75 \%$. Al instante de realizar una comparación entre cada activación independientemente, a distinta temperatura y tiempo de activación, se aprecia un mayor rendimiento en la activación química a medida que la temperatura aumenta en el menor tiempo de activación. En cambio para la activación física entre menor sea el tiempo y temperatura de activación, su rendimiento es mayor.

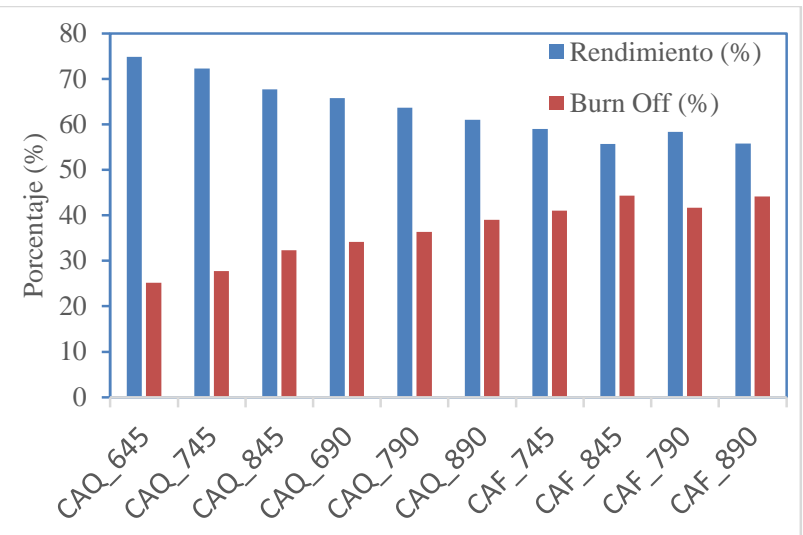

Fig. 3. Rendimiento de los carbones activados obtenidos.

Caracterización de los carbones activados y del carbón mineral: El análisis termogravimétrico del carbón mineral bituminoso se aprecia en la Fig. 4 .

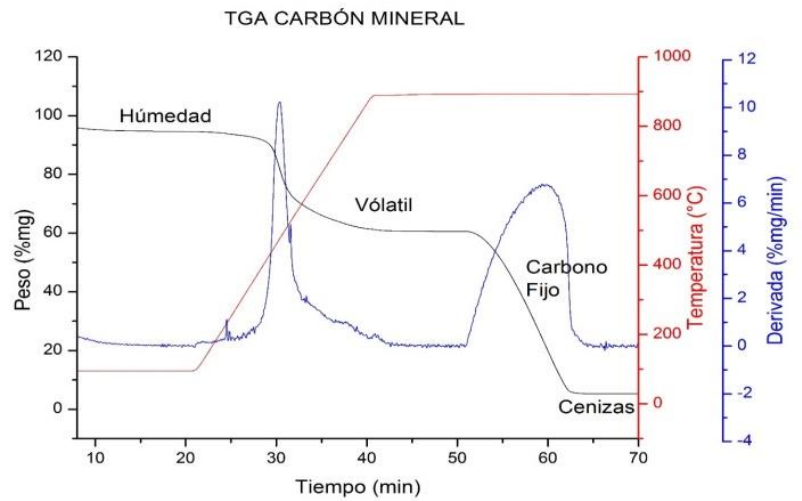

Fig. 4. Análisis termogravimétrico del carbón bituminoso de la cuenca del Cesar. 
La primera etapa del perfil termogravimétrico de la Fig 4 , a $90^{\circ} \mathrm{C}$, está asociada a la pérdida de humedad en la muestra que corresponde a un porcentaje en masa del $2.9 \%$. Alrededor de $200^{\circ} \mathrm{C}$ inicia la descomposición del material que da paso a la formación de $\mathrm{CO}, \mathrm{CO}_{2}$ y una gran variedad de compuestos volátiles.[20, 21] Alrededor de $600^{\circ} \mathrm{C}$ la mayor parte del material se ha descompuesto dejando como residuo el carbono fijo, que concierne a la organización del material carbonoso no volátil del carbón. Finalmente, cuando se cambia de una atmósfera de nitrógeno a aire se observa una última pérdida de peso, correspondiente a la combustión del carbono fijo, dejando como residuo las cenizas compuestas por el material inorgánico presente en el carbón, cuyo porcentaje en masa es del $5.59 \%$ \%.22] Los resultados del análisis elemental y próximo realizado al carbón mineral bituminoso están consignados en la Tabla 3.

Tabla 3. Propiedades del carbón mineral bituminoso. Fuente: Autores

\begin{tabular}{cccc}
\hline $\begin{array}{c}\text { Análisis } \\
\text { elemental }\end{array}$ & $\% \mathbf{p} / \mathbf{p}$ & $\begin{array}{c}\text { Análisis } \\
\text { próximo }\end{array}$ & $\% \mathbf{p} / \mathbf{p}$ \\
\hline $\mathrm{C}$ & 68.54 & Humedad & 2.93 \\
$\mathrm{~N}$ & 1.63 & Material volátil & 34.42 \\
$\mathrm{O}$ & 11.26 & Carbono fijo & 59.99 \\
$\mathrm{H}$ & 4.89 & Cenizas & 5.59 \\
$\mathrm{~S}$ & 1.12 & & \\
\hline
\end{tabular}

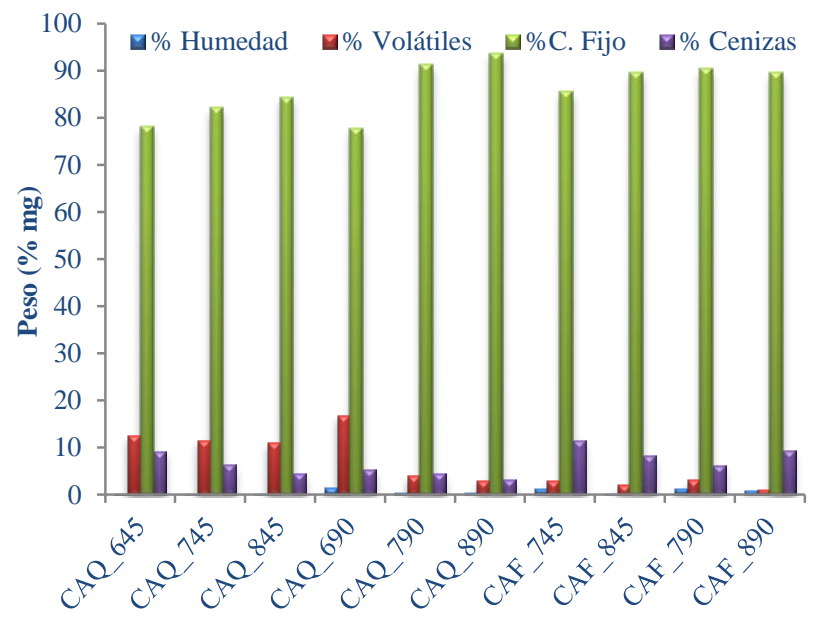

Fig. 5. Comparación de los análisis termogravimétricos de los carbones activados obtenidos.
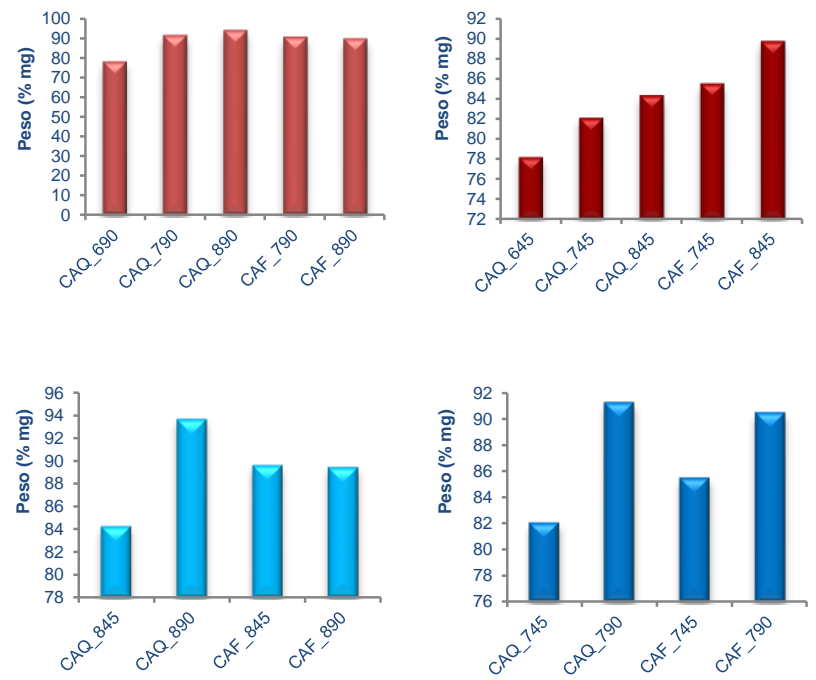

Fig. 6. Comparación del porcentaje de carbono fijo con respecto a la temperatura y tiempo de activación de los distintos carbones activados obtenidos

Al momento de comparar el porcentaje de carbono fijo para los carbones activados obtenidos en diferentes condiciones de activación, temperatura y tiempo, se aprecia mediante la Fig. 5 y 6 una variación de crecimiento porcentual. Teniendo en cuenta únicamente el método de activación se aprecia que este porcentaje de carbono fijo aumenta a medida que incrementa el tiempo y la temperatura de activación para los carbones activados químicamente; en cambio, aquellos carbones activados físicamente no se observa una gran variación pero si hay un incremento en su porcentaje de materia volátil, humedad y cenizas, que varía el porcentaje final de carbono fijo.

Con respecto al tiempo de activación el porcentaje de carbono fijo varia a los 90 minutos pero en tiempos menores se aprecia un incremento a medida que su temperatura aumenta.

La temperatura de activación es un factor importante en el cual el porcentaje de carbono fijo incrementa a menor temperatura en un tiempo de activación mayor. 


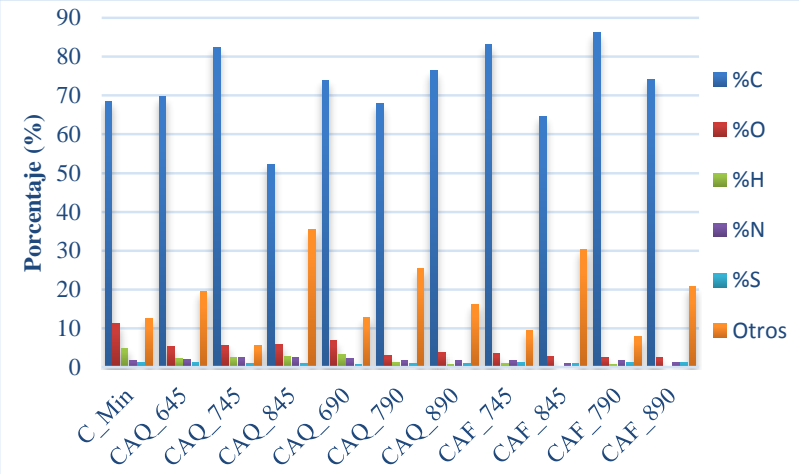

Fig. 7. Composición elemental del carbón y los distintos carbones activados obtenidos

Los resultados del análisis elemental se muestran en la Fig. 7. De acuerdo a estos resultados se puede afirmar que el proceso de pirólisis y activación en este tipo de carbón bajo las condiciones estipuladas de temperaturas y tiempos de activación, hay un mayor porcentaje de carbono en la activación física a una menor temperatura y mayor tiempo de activación, lo que indica una estructura rica en carbono. Se obtiene un menor porcentaje de oxígeno en la activación física a medida que aumenta el tiempo y la temperatura de activación. En cambio se aprecia un mayor porcentaje de hidrógeno en la activación química para los tiempos de activación menor, pero cuando este aumenta el porcentaje disminuye considerablemente. El contenido de carbono e hidrógeno permite estimar el $\mathrm{CO}_{2}$ y $\mathrm{H}_{2} \mathrm{O}$ que se produce en la combustión, normalmente estos dos compuestos se encuentran en el carbón constituyendo hidrocarburos complejos e incluso una parte de carbono se encuentra como carbonatos inorgánicos.[23, 24] El nitrógeno está confinado principalmente entre los compuestos orgánicos.[25] El contenido de azufre está relacionado con los distintos problemas medioambientales puesto que el azufre se oxida a $\mathrm{SO}_{2}$. Así un carbón con $0.9 \%$ de azufre $(22 \%$ de cenizas) provoca una concentración de $\mathrm{SO}_{2}$ en humos $\left(6 \% \mathrm{O}_{2}\right) \cdot[26]$

Tabla 4. Área superficial de los carbones activados

\begin{tabular}{cc}
\hline Muestra & Área Superficial $\left(\mathbf{m}^{\mathbf{2}} \mathbf{g}\right)$ \\
\hline CAF_890 & $351 \pm 8$ \\
CAF_790 & $46 \pm 3$ \\
CAQ_890 & $8 \pm 1$ \\
CAQ_645 & Menor a 1 \\
\hline
\end{tabular}

Los carbones activados químicamente alteraron muy poco la superficie interna del material. Esto se debe probablemente por las condiciones del tratamiento, que por las características de la muestra, parece ser que solo se activa el área superficial externa levemente, aunque se utilice la temperatura de $800^{\circ} \mathrm{C}$ y tiempos de más de una hora. En cambio los carbones activados físicamente indica que se requiere temperaturas mayores a $800^{\circ} \mathrm{C}$ para desarrollar porosidad, el cual sería recomendable para este tipo de carbón usar $900^{\circ} \mathrm{C}$. Sin embargo, la muestra CAF_890 se puede considerar un carbón activo (mirar Tabla 4), puesto que es apropiado para adsorber contaminantes en baja concentración en fase gaseosa y algunos metales 0 compuestos orgánicos en fase líquida, además que el material producido sería de bajo costo y el tiempo de saturación sería bajo lo cual aumentaría así el número de regeneraciones.[27]

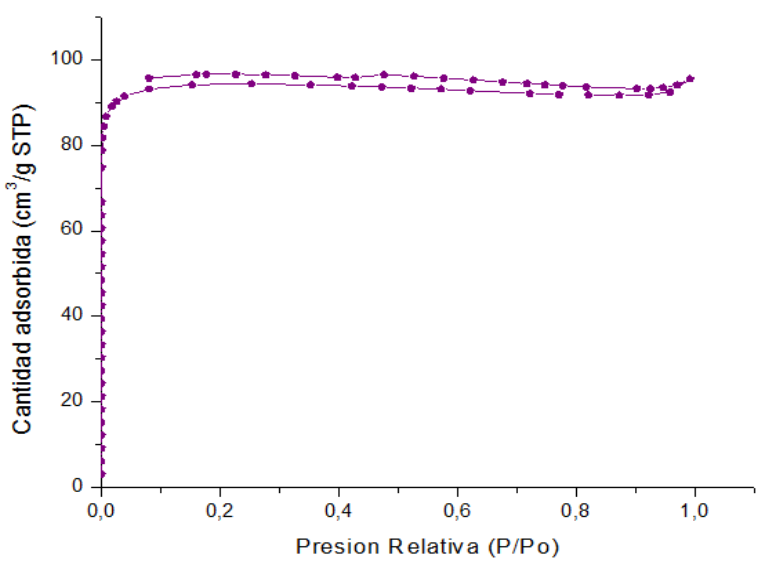

Fig. 8. Isoterma de adsorción y desorción de nitrógeno a 77K para el CAF_890.

Las isotermas obtenidas son de tipo I de acuerdo con la clasificación de Brunauer, como se aprecia en la Fig. 8. Esta se caracteriza por presentar un fuerte aumento en la región de bajas presiones y un plato en la región de altas presiones que indican la naturaleza microporosa de los carbones.[28]

Este tipo de isoterma indica que el proceso de llenado de microporosos se produce a bajas presiones relativas, sin contribución de poros de mayor tamaño, lo que indica una estrecha distribución de tamaño microporoso, además esta isoterma presenta una meseta horizontal y un ciclo de histéresis apenas visible.[29] 
En la Fig. 9 se muestra una micrografía SEM del carbón activado CAF_890, en la que se aprecia la formación de pequeños aglomerados y zonas de bajo contenido mineral que se caracterizan por la presencia de $\mathrm{Al}, \mathrm{Si}, \mathrm{Fe}, \mathrm{Zn}, \mathrm{Cl}, \mathrm{K}$ y O; es decir, aparte de la presencia de carbono también se tienen aluminosilicatos y hierro propios de la materia mineral del carbón como se observa en el análisis elemental de la superficie EDX del carbón activado CAF_890, Fig. 10.[30]

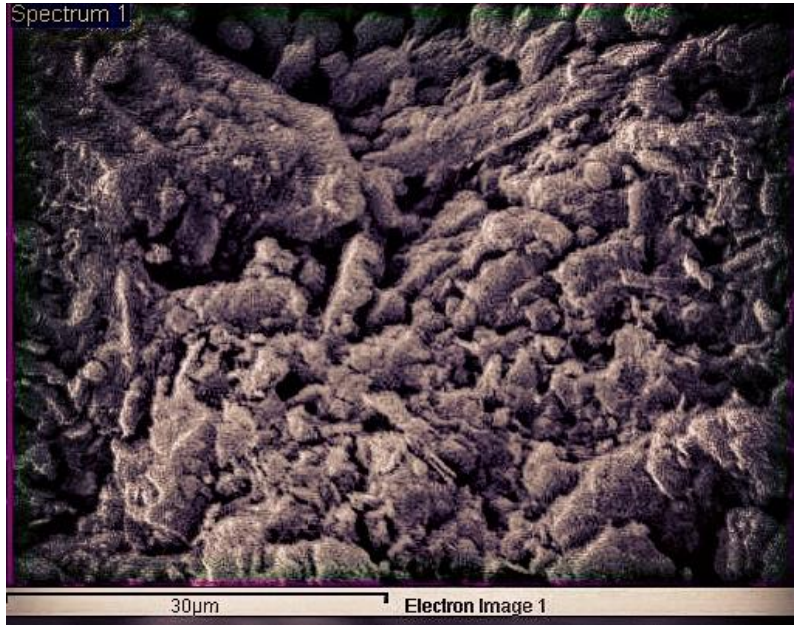

Fig. 9. Micrografía SEM para el CAF_890.

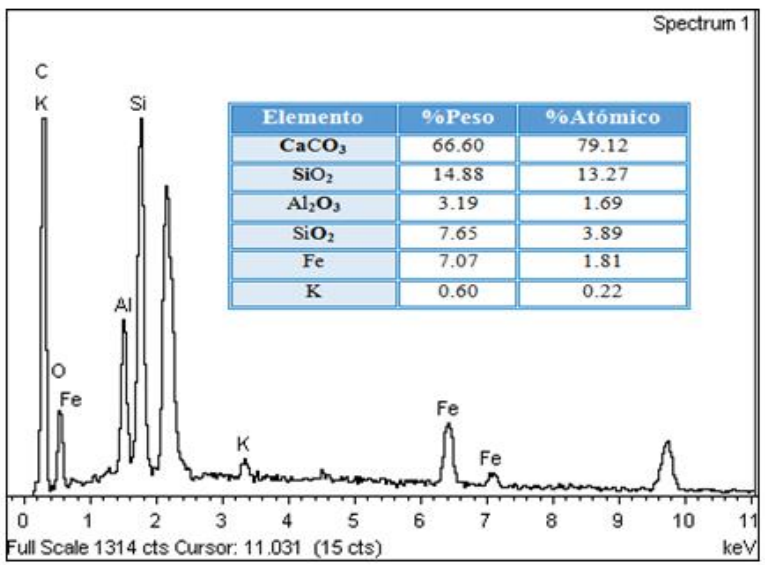

Fig. 10. Análisis elemental en la superficie EDX para el CAF_890.

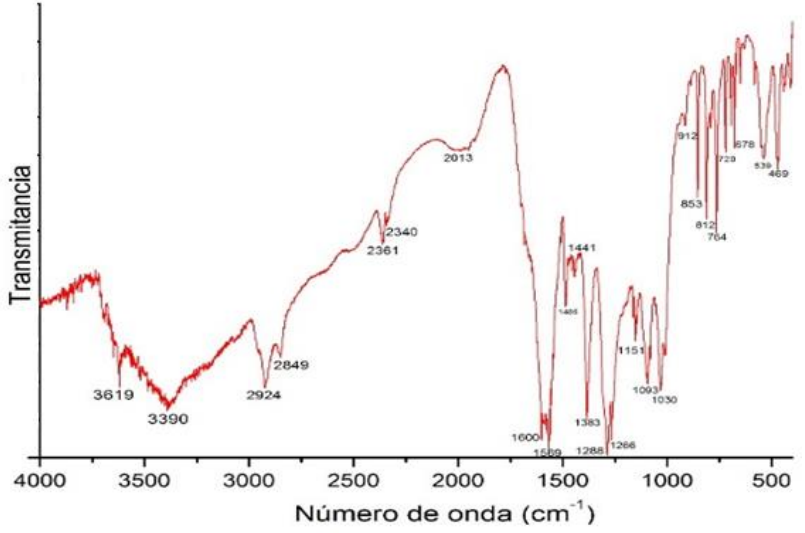

Fig. 11. Espectro FTIR del carbón mineral bituminoso.

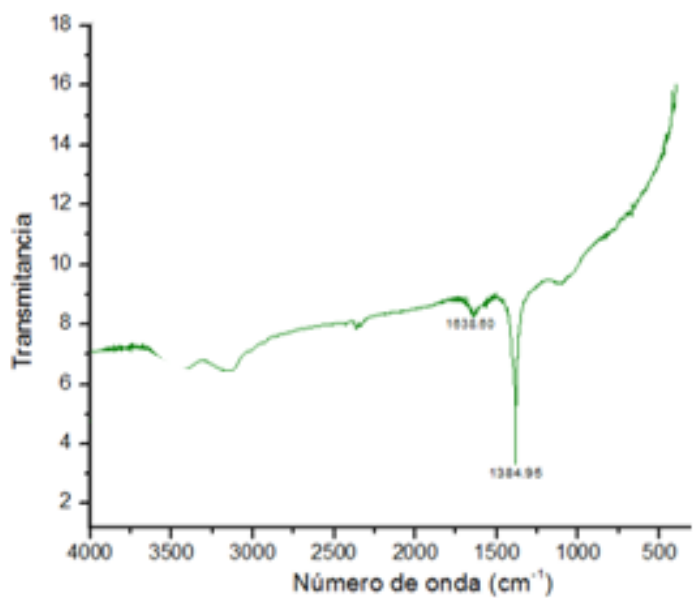

Fig. 12. Espectro FTIR del carbón activado CAF 890.

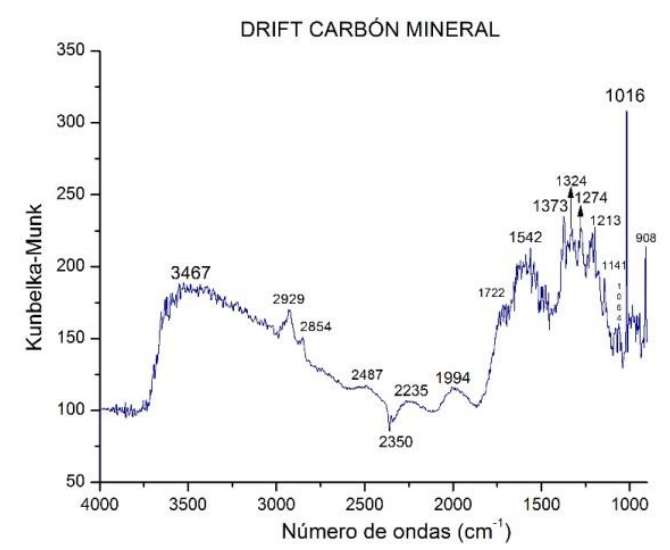

Fig. 13. Espectro DRIFT del carbón mineral bituminoso. 


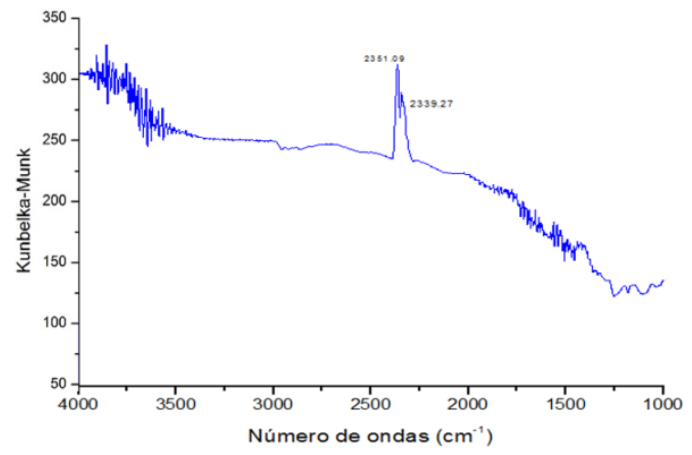

Fig. 14. Espectro DRIFT del carbón activado CAF_890.

La espectroscopia FTIR \& DRIFT se aprecian en las Fig. 11, 12, 13 y 14.

La estructura del carbón mineral durante la carbonización y pirólisis, favorece a la conversión de compuestos aromáticos.

En la activación física se observa una disminución de estas bandas específicamente en el área 35003000 , las cuales disminuye su intensidad que puede deberse al rompimiento de los enlaces característicos y la desintegración de las especies que no soportan la temperatura de pirolisis. Sin embargo, cabe destacar que a medida que incrementa la temperatura de los carbones activados en estudio, se aprecia una notoria disminución de las señales e intensidad de los picos posiblemente por la pérdida de productos volátiles $\left(\mathrm{CO}, \mathrm{CO}_{2}, \mathrm{CH}_{4}\right)$.[31] Estos resultados podrían estar ligados con la naturaleza ácido-base de los carbones y su presencia o ausencia de oxígeno. Es decir, las bandas de absorción en el infrarrojo de los carbones puede deberse a la presencia de electrones $\pi$ deslocalizados en la superficie de los carbones y a estructuras aromáticas.[32]

El punto de carga cero (PZC) es el valor de $\mathrm{pH}$ donde la carga superficial neta del adsorbente es cero; es decir, que las concentraciones de $\mathrm{H}^{+}$y $\mathrm{OH}^{-}$ adsorbidos sobre la superficie deben ser iguales, siendo así que la carga superficial en ese instante es neutra como se observa en la Fig. 15.[33]

En el caso del CAF_890 arrojo un valor igual a 5.6, esto nos indica que las condiciones más apropiadas para que este adsorba eficientemente compuestos catiónicos como azul de metileno es a $\mathrm{pH}$ superiores $\mathrm{PZC}$, ya que posee una superficie de carga negativa por lo tanto se verá favorecida la adsorción de colorantes catiónicos.[34]

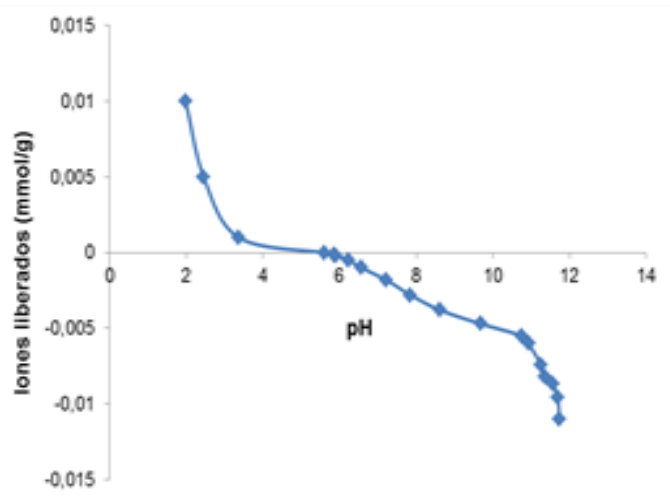

Fig. 15. Punto de carga cero para el carbón activado CAF_890

La adsorción del colorante de azul de metileno sobre el carbón activado físicamente, se estudió empleando los modelos de Langmuir y Freundlich como se aprecia en las siguientes figuras (Fig. 16, 17 y 18).

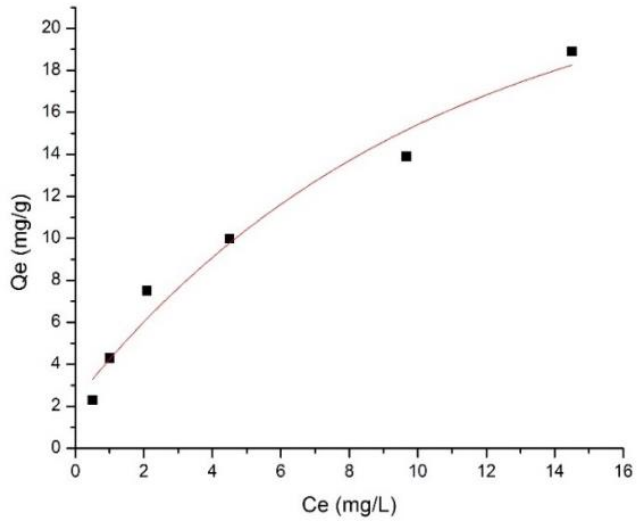

Fig. 16. Isoterma de adsorción de azul de metileno.

Para el modelo de Langmuir se empleó la ecuación (2) con su respectiva linealización en la ecuación (3), cuyos valores se encuentran arrojados en la Tabla 5.

$$
\begin{aligned}
& q_{e}=\frac{q_{\max } \times b \times C_{e q}}{1+b \times C_{e q}} \\
& \frac{1}{q_{e}}=\frac{1}{Q \max }+\left(\frac{1}{b Q \max } \times \frac{1}{C e q}\right)
\end{aligned}
$$




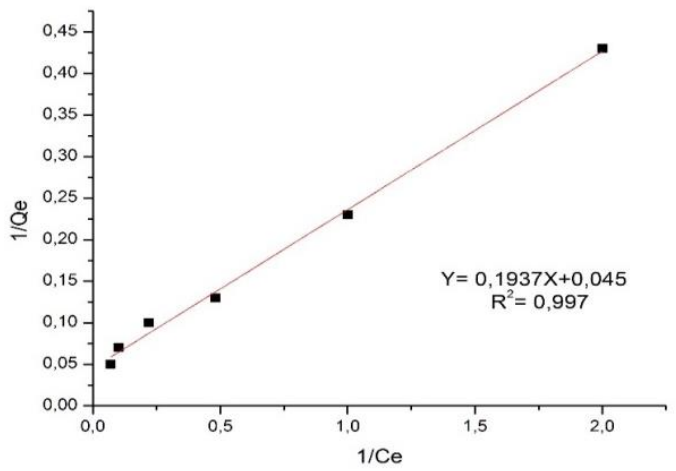

Fig. 17. Linealización de la isoterma de Langmuir para el azul de metileno.

De igual forma se emplearon las ecuaciones (4) y (5) para Freundlich, cuyos valores obtenidos se aprecian en la Tabla 5.

$$
q_{e}=K f C_{e q}^{1 / n}
$$

$$
\log q_{e}=\log K f+\frac{1}{n} \log C e q
$$

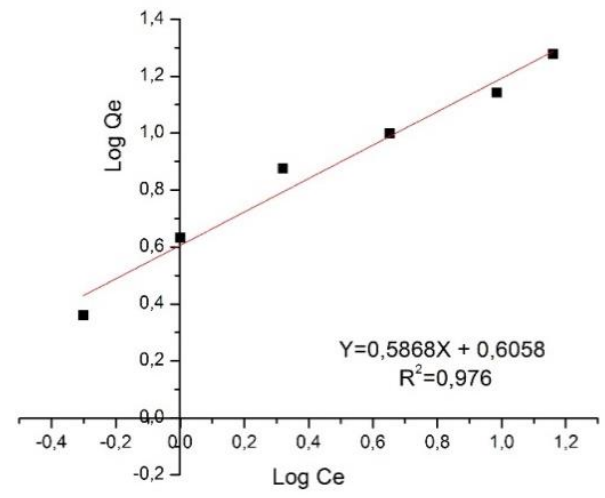

Fig. 18. Linealización de la isoterma de Freundlich para el azul de metileno

Tabla 5. Constante de adsorción para la isoterma de Langmuir. Y Freundlich.

Isoterma de Langmuir

\begin{tabular}{llll}
\hline Qmax (mg/g) & $\mathbf{b}(\mathbf{L} / \mathbf{m g})$ & $\mathbf{R}^{\mathbf{2}}$ \\
\hline 34.46 & 0.1503 & 0.997 \\
\hline \multicolumn{4}{c}{} \\
\hline $\mathbf{K}$ & Isoterma Freundlich \\
\hline 3.93 & $\mathbf{n}$ & $\mathbf{R}^{\mathbf{2}}$ \\
\hline
\end{tabular}

La adsorción de azul de metileno en el carbón activado físicamente CAF_890 presentó una mayor afinidad por la ecuación de la isoterma de Langmuir $\left(R^{2}=0,997\right)$, Fig. 17 , lo cual permite predecir que prevalece el proceso de cobertura de la monocapa, con una máxima capacidad de la monocapa (Qmax) obtenida fue de 34,46 mg/g para el carbón activado. Sin embargo, se puede decir que la capacidad de adsorción se encuentra influenciada por factores como la estructura química del colorante, la química superficial del carbón activado, el tamaño de partícula, entre otros.[35]

De acuerdo con los resultados obtenidos el carbón activado se podría emplear como un adsorbente efectivo y considerarse como una alternativa para la remoción de colorantes.

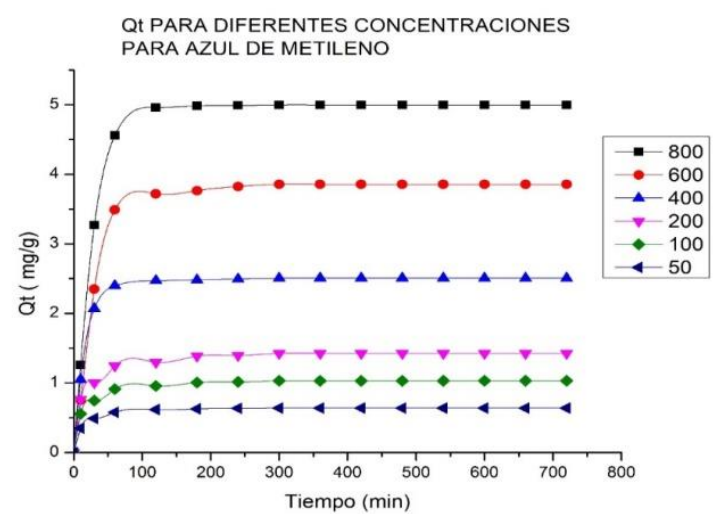

Fig. 19. Capacidad de adsorción para distintas concentraciones de azul de metileno en el carbón activado CAF_890.

El estudio cinético permite establecer la velocidad a la que los contaminantes, en este caso, los colorantes, son removidos de la fase acuosa. La remoción es una función del tiempo de contacto, en el cual se puede apreciar que a medida que aumentan las diferentes concentraciones iniciales con respecto al tiempo de contacto los valores de Qt van incrementando simultáneamente. Para un tiempo máximo de 12 horas y al incrementar la concentración inicial del azul de metileno de 50 $\mathrm{mg} / \mathrm{L}$ a $800 \mathrm{mg} / \mathrm{L}$, permite que el carbón activado empleado como adsorbente aumente su capacidad de adsorción, como se aprecia en la Figura 19. Además se observa que la adsorción del carbón activado ocurre inicialmente de forma rápida en los primeros 100 minutos, luego esta adsorción se lleva por un proceso más lenta.[36-39] Este estudio cinético fue llevado a cabo con tres modelos cinéticos: Modelo cinético Pseudo Primer y 
Segundo orden de Largegren (Fig. 20-21, Ecuaciones 6-7, Tabla 6-7) y el Modelo de Difusión intraparticular de Weber \& Morris (Fig. 22, Tabla 8, Ecuación 8).

Ecuación del modelo pseudo-primer orden:

$$
\log \left(\frac{q e}{q e-q t}\right)=\frac{K_{1} t}{2,303}
$$

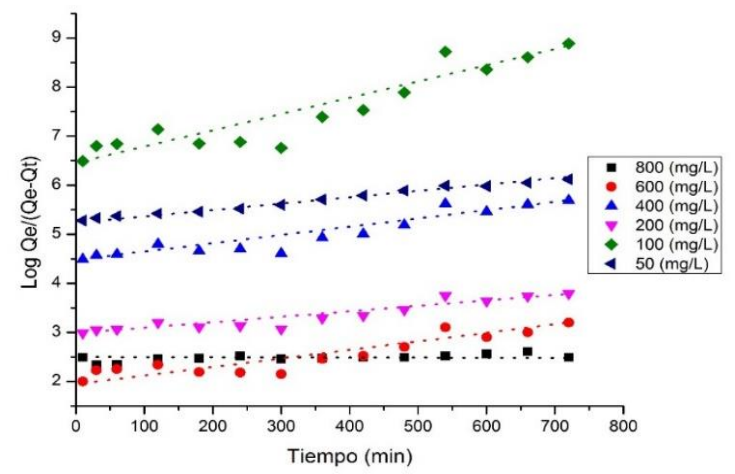

Fig. 20. Modelo pseudo-primer orden para distintas concentraciones de azul de metileno en el carbón activado CAF_890

Tabla 6. Valores $\mathrm{R}^{2}$

\begin{tabular}{cc}
\hline Concentraciones $\mathbf{( m g / L )}$ & $\mathbf{R}^{\mathbf{2}}$ \\
\hline 50 & 0.9889 \\
100 & 0.8666 \\
200 & 0.8935 \\
400 & 0.8855 \\
600 & 0.8372 \\
800 & 0.4981 \\
\hline
\end{tabular}

Ecuación del modelo pseudo-segundo orden:

$$
\frac{t}{q t}=\frac{1}{K_{2}^{\prime} q_{e}^{2}}+\frac{1}{q e} t
$$

Al comparar los valores de $\mathrm{R}^{2}$ obtenidos de los modelos cinéticos de Largergren en la adsorción de azul de metileno evaluado a distintas concentraciones, se apreció que el modelo al que se le ajusta es pseudo-segundo orden, puesto que el coeficiente de correlación arrojado es de 0,9999 aproximadamente 1 .
Ecuación del modelo de difusión intraparticular de Weber \& Morris.

$$
q=K_{p} t^{0,5}
$$

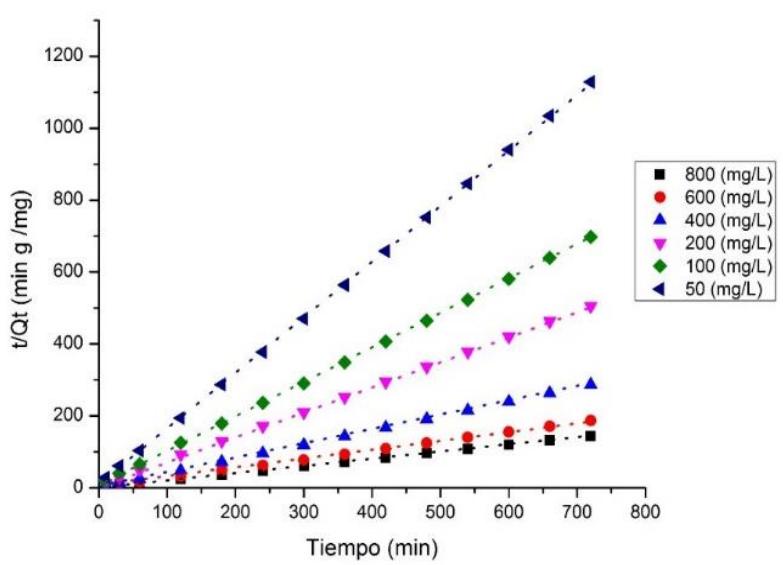

Fig. 21. Modelo pseudo-segundo orden para distintas concentraciones de azul de metileno en el carbón activado CAF_890

Tabla 7. Valores $\mathrm{R}^{2}$

\begin{tabular}{cc}
\hline Concentraciones $\mathbf{( m g / L )}$ & $\mathbf{R}^{\mathbf{2}}$ \\
\hline 50 & 0.9999 \\
100 & 0.9999 \\
200 & 0.9999 \\
400 & 0.9999 \\
600 & 0.9992 \\
800 & 0.9985 \\
\hline
\end{tabular}

Los valores obtenidos de $\mathrm{R}^{2}$ para el azul de metileno en diferentes concentraciones se encuentra dentro de un rango $0,95-0,99$, esto nos da a sugerir que la adsorción podría estar determinada por un modelo de difusión intraparticular, además se aprecia que los puntos no pasan del origen, lo que indica que la difusión no es el único mecanismo que controla la velocidad de adsorción sino que además esta descrita por el modelo cinético pseudo segundo orden.[40, 41] 


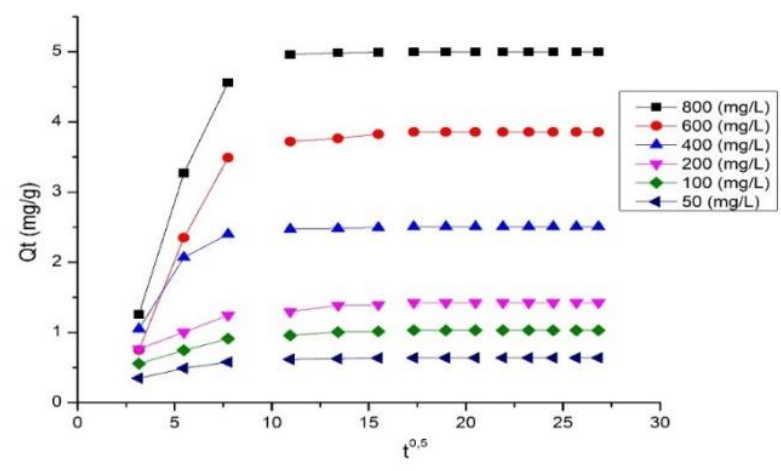

Fig. 22. Modelo Weber-Morris difusión intraparticular para el carbón activado CAF 890

Tabla 8. Parámetros de difusión intrapartícular para el azul de metileno.

\begin{tabular}{lll}
\hline $\begin{array}{l}\text { Concentraciones } \\
(\mathbf{m g} / \mathbf{L})\end{array}$ & $\begin{array}{l}\mathrm{Kp} \\
\left(\mathbf{m g} / \mathbf{g m i n}^{-1}\right)\end{array}$ & $\mathbf{R}^{\mathbf{2}}$ \\
\hline 50 & 0.0152 & 0.98 \\
100 & 0.0256 & 0.99 \\
200 & 0.0359 & 0.97 \\
400 & 0.0611 & 0.96 \\
600 & 0.1148 & 0.94 \\
800 & 0.1419 & 0.95 \\
\hline
\end{tabular}

Esto lo que revela es que posiblemente ocurre un proceso de quimisorción e intercambio iónico entre el azul de metileno y los grupos funcionales presentes en el carbón activado.[42, 43] A su vez se observa que a medida que incrementa la concentración aumenta los valores Kp; es decir, en este proceso puede estar ocurriendo un incremento de la fuerza de difusión debido al ascenso de la concentración, la adsorción que pudo haber ocurrido en los poros o en la superficie del material, teniendo en cuenta que el tamaño de la molécula es de más de $13 \AA ̊$ de largo $(>1.3 \mathrm{~nm})$ y más de $5 \AA ̊$ de ancho $(>0.5 \mathrm{~nm})$, lo cual es muy probable que la adsorción se esté dando en algunos de los microporos desarrollados.[44]

\section{CONCLUSIONES}

Se obtuvieron carbones activados con áreas superficiales de $351 \mathrm{~m}^{2} / \mathrm{g}$ para CAF_890, $46 \mathrm{~m}^{2} / \mathrm{g}$ para CAF_790, $8 \mathrm{~m}^{2} / \mathrm{g}$ para $\mathrm{CAQ} \_890$ y menores de $1 \mathrm{~m}^{2} / \mathrm{g}$ para CAQ_645. Lo cual indica que las condiciones de activación química usadas no son apropiadas para este tipo de material.

El carbón activado con mayor área superficial (CAF_890), presentó una microporosidad con un volumen de poro de $0.15 \mathrm{~cm}^{3} / \mathrm{g}$ y un tamaño promedio de poro de $19.40 \AA$. El punto de carga cero del carbón activado CAF 890 fue de 5.6, lo que indica que el carbón activado posee carga superficial negativa, por lo tanto la solución de azul de metileno fue a $\mathrm{pH}$ mayores que este punto.

El carbón activado CAF_890 fue apto para la remoción del azul de metileno. La isoterma de adsorción se ajustó al modelo propuesto por Langmuir y la cinética de adsorción se ajustaron al modelo pseudo-segundo orden y de difusión intraparticular, lo que establece que la adsorción no se dio por un solo mecanismo de acuerdo al control de la velocidad de adsorción.

\section{AGRADECIMIENTOS}

Los autores agradecen al Grupo de investigación de Fotoquímica y Fotobiologia (GFF) del programa de Química, al Grupo de Carboquímica de la Facultad de Ingeniería de la Universidad del Atlántico. El Grupo de Química de Recursos Energéticos y Medio Ambiente (QUIREMA) agradece al Programa de Sostenibilidad de la Universidad de Antioquia.

\section{REFERENCIAS BIBLIOGRÁFICAS}

[1] Mohan D, Singh KP, Singh VK. Wastewater treatment using low cost activated carbons derived from agricultural byproducts-A case study. Journal of Hazardous Materials. 152, 1045-1053, 2008.

[2] Faur-Brasquet C, Kadirvelu K, Le Cloirec P. Removal of metal ions from aqueous solution by adsorption onto activated carbon cloths: adsorption competition with organic matter. Carbon. 40, 23872392, 2002.

[3] Guedidi H, Reinert L, Soneda Y, Bellakhal N, Duclaux L. Adsorption of ibuprofen from aqueous solution on chemically surface-modified activated carbon cloths. Arabian Journal of Chemistry. 10, S3584-S3594, 2017. 
[4] Freeman JJ. Active carbon. Journal of Chemical Technology \& Biotechnology. 48, 240-241, 1990.

[5] Özkaya B. Adsorption and desorption of phenol on activated carbon and a comparison of isotherm models. Journal of Hazardous Materials. 129, 158163, 2006.

[6] Yu S, Wang X, Yao W, Wang J, Ji Y, Ai Y, et al. Macroscopic, Spectroscopic, and Theoretical Investigation for the Interaction of Phenol and Naphthol on Reduced Graphene Oxide. Environmental Science \& Technology. 51, 32783286, 2017.

[7] Blanco-Martínez DA, Giraldo L, Moreno-Piraján JC. Effect of the $\mathrm{pH}$ in the adsorption and in the immersion enthalpy of monohydroxylated phenols from aqueous solutions on activated carbons. Journal of Hazardous Materials. 169, 291-296, 2009.

[8] Peña K, Giraldo L, Moreno JC. Preparación de carbón activado a partir de cáscara de naranja por activación química. Caracterización física y química. Revista Colombiana de Química. 41, 311323, 2012.

[9] Moreno-Castilla C. Adsorption of organic molecules from aqueous solutions on carbon materials. Carbon. 42, 83-94, 2004.

[10] Tonucci MC, Gurgel LVA, Aquino SFd. Activated carbons from agricultural byproducts (pine tree and coconut shell), coal, and carbon nanotubes as adsorbents for removal of sulfamethoxazole from spiked aqueous solutions: Kinetic and thermodynamic studies. Industrial Crops and Products. 74, 111-121, 2015.

[11] Rakić V, Rac V, Krmar M, Otman O, Auroux A. The adsorption of pharmaceutically active compounds from aqueous solutions onto activated carbons. Journal of Hazardous Materials. 282, 141149, 2015.

[12] de Sousa JC, Parra JB, Pajares JA, Pis JJ. Activated carbons from semianthracite by steam activation. Effect of coal preoxidation and burn-off. Studies in Surface Science and Catalysis. 87, 603612, 1994.
[13] Larsen JW, Green TK, Kovac J. The nature of the macromolecular network structure of bituminous coals. The Journal of Organic Chemistry. 50, 47294735, 1985.

[14] Derbyshire F. Vitrinite structure: alterations with rank and processing. Fuel. 70, 276-284, 1991.

[15] Haenel MW. Recent progress in coal structure research. Fuel. 71, 1211-1223, 1992.

[16] Marzec A, Kisielow W. Mechanism of swelling and extraction and coal structure. Fuel. 62, 977979, 1983.

[17] Shinn JH. From coal to single-stage and twostage products: A reactive model of coal structure. Fuel. 63, 1187-1196, 1984.

[18] Solache-Ríos MJ, Villalva-Coyote R, Díaz-Nava MdC. Sorption and Desorption of Remazol Yellow by a Fe-Zeolitic Tuff. Journal of the Mexican Chemical Society. 54, 59-68, 2010.

[19] Bofan G, Weizhuang H, Qixiu Z. A study on separation of molybdenum from tungsten by an adsorption process using activated carbon. International Journal of Refractory Metals and Hard Materials. 14, 319-323, 1996.

[20] Sui Q, Huang J, Liu Y, Chang X, Ji G, Deng S, et al. Rapid removal of bisphenol $A$ on highly ordered mesoporous carbon. Journal of Environmental Sciences. 23, 177-182, 2011.

[21] Zhang L, Xu T, Liu X, Zhang $\mathrm{Y}$, Jin $\mathrm{H}$. Adsorption behavior of multi-walled carbon nanotubes for the removal of olaquindox from aqueous solutions. Journal of Hazardous Materials. 197, 389-396, 2011.

[22] Cao N, Darmstadt H, Soutric F, Roy C. Thermogravimetric study on the steam activation of charcoals obtained by vacuum and atmospheric pyrolysis of softwood bark residues. Carbon. 40, 471-479, 2002.

[23] Bandosz TJ, Ania CO. Chapter 4 Surface chemistry of activated carbons and its characterization. Interface Science and Technology. 7, 159-229, 2006. 
[24] Menéndez-Díaz JA, Martín-Gullón I. Chapter 1 Types of carbon adsorbents and their production. Interface Science and Technology. 7, 1-47, 2006.

[25] Guo P, Gu Y, Lei Z, Cui Y, Zhao XS. Preparation of sucrose-based microporous carbons and their application as electrode materials for supercapacitors. Microporous and Mesoporous Materials. 156, 176-180, 2012.

[26] Helmich M, Luckas M, Pasel C, Bathen D. Characterization of microporous activated carbons using molecular probe method. Carbon. 74, 22-31, 2014.

[27] Gokulakrishnan N, Pandurangan A, Sinha PK. Removal of Decontaminating Agent from Aqueous Solution Using Microporous and Mesoporous Materials: Activated Carbon as an Effective Adsorbent. Adsorption Science \& Technology. 26, 291-302, 2008.

[28] Zhu G-Z, Deng X-I, Hou M, Sun K, Zhang Y-p, $\mathrm{Li} P$, et al. Comparative study on characterization and adsorption properties of activated carbons by phosphoric acid activation from corncob and its acid and alkaline hydrolysis residues. Fuel Processing Technology. 144, 255-261, 2016.

[29] Atkinson JD, Rood MJ. Preparing microporous carbon from solid organic salt precursors using in situ templating and a fixed-bed reactor. Microporous and Mesoporous Materials. 160, 174-181, 2012.

[30] Laine J, Calafat A, labady M. Preparation and characterization of activated carbons from coconut shell impregnated with phosphoric acid. Carbon. 27, 191-195, 1989.

[31] Arampatzidou AC, Deliyanni EA. Comparison of activation media and pyrolysis temperature for activated carbons development by pyrolysis of potato peels for effective adsorption of endocrine disruptor bisphenol-A. Journal of Colloid and Interface Science. 466, 101-112, 2016.

[32] Laginhas C, Nabais JMV, Titirici MM. Activated carbons with high nitrogen content by a combination of hydrothermal carbonization with activation. Microporous and Mesoporous Materials. 226, 125132, 2016.
[33] Yan XB, Xu T, Chen G, Liu HW, Yang SR. Effect of deposition voltage on the microstructure of electrochemically deposited hydrogenated amorphous carbon films. Carbon. 42, 3103-3108, 2004.

[34] Sugashini S, Begum KMMS. Preparation of activated carbon from carbonized rice husk by ozone activation for $\mathrm{Cr}(\mathrm{VI})$ removal. New Carbon Materials. 30, 252-261, 2015.

[35] Díaz-Faes E, Barriocanal C, Díez MA, Alvarez R. Applying TGA parameters in coke quality prediction models. Journal of Analytical and Applied Pyrolysis. 79, 154-160, 2007.

[36] Larsen JW, Gorbaty ML. Coal Structure and Reactivity A2. In: Meyers R, editor. Encyclopedia of Physical Science and Technology (Third Edition). New York: Academic Press, 107-122, 2003.

[37] Solomon PR, Serio MA, Suuberg EM. Coal pyrolysis: Experiments, kinetic rates and mechanisms. Progress in Energy and Combustion Science. 18, 133-220, 1992.

[38] Varma AK. Thermogravimetric investigations in prediction of coking behaviour and coke properties derived from inertinite rich coals. Fuel. 81, 13211334, 2002.

[39] Molina-Sabio M, RodRíguez-Reinoso F, Caturla $F$, Sellés MJ. Porosity in granular carbons activated with phosphoric acid. Carbon. 33, 1105-1113, 1995.

[40] López-González JdD, Moreno-Castilla C, Rodriguez-Ramos I, Rodriguez-Reinoso F. 177. The use of activated carbons as supports for platinum catalysts. Carbon. 22, 224, 1984.

[41] Rodríguez-Reinoso F. Activated Carbon and Adsorption A2 - Buschow, K.H. Jürgen. In: Cahn RW, Flemings MC, Ilschner B, Kramer EJ, Mahajan $S$, Veyssière $P$, editors. Encyclopedia of Materials: Science and Technology (Second Edition). Oxford: Elsevier, 22-34, 2001.

[42] Alcañiz-Monge J, Marco-Lozar JP, LozanoCastelló D. Monolithic Carbon Molecular Sieves from activated bituminous coal impregnated with a slurry of coal tar pitch. Fuel Processing Technology. 95, 67-72, 2012. 
[43] Azizian S, Haerifar M, Bashiri H. Adsorption of methyl violet onto granular activated carbon: Equilibrium, kinetics and modeling. Chemical Engineering Journal. 146, 36-41, 2009.

[44] Cheung WH, Szeto YS, McKay G. Intraparticle diffusion processes during acid dye adsorption onto chitosan. Bioresource Technology. 98, 2897-2904, 2007. 